\title{
SCIENTIFIC REPORTS

\section{Red blood cell distribution width predicts long-term mortality in critically ill patients with acute kidney injury: a retrospective database study}

\author{
Linpei Jia ${ }^{1,7^{*}}$, Shijun Cui ${ }^{2,7}$, Jingyan Yang ${ }^{3}$, Qiang $\mathrm{Jia}^{1}$, Lixiao Hao ${ }^{4,5}$, Rufu $\mathrm{Jia}^{3 *} \&$ \\ Hongliang Zhang ${ }^{6 *}$
}

Acute kidney injury (AKI) is a serious complication in the intensive care unit (ICU), which may increase the mortality of critically ill patients. The red blood cell distribution width (RDW) has proved useful as a predictor of short-term prognosis in critically ill patients with AKI. However, it remains unknown whether RDW has a prognostic value of long-term all-cause mortality in these patients. The data of 18279 critically ill patients with AKI at first-time hospital admission were extracted from the Medical Information Mart for Intensive Care III (MIMIC-III) database. The tertiles of the RDW values were used to divide subjects into three groups, namely RDW $<13.6 \%$ for the low RDW group, $13.6 \% \leq \mathrm{RDW}<15.2 \%$ for the middle RDW group and RDW $\geq 15.2 \%$ for the high RDW group. Demographic data, mortality, 4-year survival time and severity scale scores were compared among groups. The Kaplan-Meier analysis and the Cox regression analysis were performed to assess the impact of RDW on all-cause mortality in AKI patients. The receiver operating characteristic $(R O C)$ curve analysis was done to evaluate the prognostic value of RDW on the long-term outcome of critically ill patients with AKI. The median age of the enrolled subjects was 65.6 years. AKI patients with a higher RDW value had significantly shorter survival time and higher death rate. By the Kaplan-Meier analysis, patients in the higher RDW group presented significantly shorter survival time and higher death rate. The Cox regression model indicated RDW as an independent risk factor of all-cause mortality of AKI patients (HR 1.219, 95\% Cl, 1.211 to 1.228). By the ROC analysis, RDW appeared more efficient in predicting long-term prognosis as compared with conventional severity scales. The AUC of RDW $(95 \% \mathrm{Cl}, 0.712$ to 0.725$)$ was significantly higher than other severity scale scores. In conclusion, RDW is positively correlated to survival time of 4-year follow-up in critically ill patients with AKI, and RDW is an independent prognostic factor of longterm outcomes of these patients.

Acute kidney injury (AKI) is one of the most common complications in critically ill patients, which results in poor prognosis and high risk of death ${ }^{1}$. Approximately $57 \%$ of patients in the intensive care unit (ICU) were complicated with AKI, and up to $27 \%$ died consequently during hospitalization ${ }^{2}$. When AKI occurs concomitantly with other severe organ dysfunctions like myocardial infarction or sepsis, the mortality rate is further increased to $45 \%$ to $60 \%{ }^{3}$.

\footnotetext{
${ }^{1}$ Department of Nephrology, Xuanwu Hospital, Capital Medical University, Changchun Street 45\#, 100053, Beijing, China. ${ }^{2}$ Department of Vascular Surgery, Xuanwu Hospital, Capital Medical University, Changchun Street 45\#, 100053, Beijing, China. ${ }^{3}$ Central Hospital of Cangzhou, Xinhua Middle Street 201\#, 061001, Cangzhou, Hebei Province, China. ${ }^{4}$ Department of Gerontology, Xuanwu Hospital, Capital Medical University, Changchun Street 45\#, 100053, Beijing, China. ${ }^{5}$ Department of General Medicine, Xuanwu Hospital, Capital Medical University, Changchun Street 45\#, 100053, Beijing, China. ${ }^{6}$ Department of Life Sciences, National Natural Science Foundation of China, Shuangqing Road 83\#, 100085, Beijing, China. ${ }^{7}$ These authors contributed equally: Linpei Jia and Shijun Cui. *email: anny_069@163.com; zxyy5688@126.com; drzhl@hotmail.com
} 
A number of scoring systems are being used to evaluate severity and to predict prognosis of critically ill patients, but none of them is specific for those patients complicated with AKI. Moreover, although various biomarkers are frequently used to predict short-term prognosis and in-hospital mortality of critically ill patients with AKI, including urinary neutrophil gelatinase-associated lipocalin ${ }^{4}$, right ventricular longitudinal strain ${ }^{5}$, microRNAs ${ }^{6}$ and so forth, long-term prognosis is scarcely evaluated due to the difficulty in long-term follows-up. Since AKI may result in permanent injuries of kidney and the long-term risk of death is twice as high in patients with $\mathrm{AKI}$ as those without $\mathrm{AKI}^{7}$, prediction of long-term prognosis and early intervention for AKI patients are of utmost importance.

The red blood cell distribution width (RDW) represents the variability in size of circulating erythrocytes ${ }^{8}$. Clinically, RDW is useful for the prognostic prediction of acute disease like sepsis ${ }^{9}$ and pancreatitis ${ }^{10}$. Although several studies reported the prognostic efficiency of RDW on AKI, conclusions are inconsistent. Wang et al. reported that the short-term prognostic value of RDW was more accurate than Acute Physiology Score III (APS III) and the Sequential Organ Failure Assessment (SOFA) in critically ill patients with $\mathrm{AKI}^{11}$. Hu et al. also demonstrated that RDW was an independent predictor for AKI and mortality in patients in the coronary care unit $^{8}$. Instead, Elhosseiny et al. found that RDW did not correlate with the development of contrast-induced $\mathrm{AKI}^{12}$. Besides, all previous studies were focused on the short-term prediction efficiency of RDW on AKI. Whether RDW can be used to predict the long-term prognosis in AKI patients and the efficiency of RDW therein as compared with conventional scales merit further investigation.

In this study, data of critically ill patients complicated with AKI during 48 hours after admission to ICU were extracted. We hypothesized that RDW is useful in the prediction of long-term prognosis of critically ill patients with AKI. We analyzed the relationship between RDW and the survival time of patients at different AKI stages. The prognostic value of 4 -year mortality as measured by RDW and/or RDW combined with several severity scales was evaluated.

\section{Subjects and methods}

Data source. Data were extracted from the Medical Information Mart for Intensive Care III (MIMIC-III) database, which recorded the demographic data, vital signs, medications and other important items of 53,423 adult admissions to ICUs in the Beth Israel Deaconess Medical Center in Boston from 2001 to $2012^{13}$. The establishment of the MIMIC-III database was approved by the Massachusetts Institute of Technology and the Institutional Review Boards. According to the Health Insurance Portability and Accountability Act (HIPAA) standards (www.hhs.gov), eighteen identifying data elements were removed from the MIMIC-III database ${ }^{13}$. Our study was conducted entirely on publicly available, anonymized data, thus individual patient consents were waived. To get the permission of access to the MIMIC-III database, Linpei Jia passed the Protecting Human Research Participants Exam of National Institutes of Health (Record ID: 27638410).

Inclusion and exclusion criteria. AKI was defined by the Acute Kidney Injury Network (AKIN) criteria, i.e. serum creatinine (Scr) values increasing $\geq 26.5 \mu \mathrm{mol} / \mathrm{L}$ or 1.5 -fold of baseline values during 48 hours, or urine out $<0.5 \mathrm{~mL} /(\mathrm{kg} \cdot \mathrm{h})$ for more than 6 hours $^{14}$. In our study, we used the AKIN criteria instead of the Kidney Disease: Improving Global Outcomes (KDIGO) criteria for two reasons. One reason is that most data in the MIMIC-III database were recorded before the publication of the KDIGO guideline ${ }^{15}$. Likewise, some other studies used the AKIN criteria for the same reason ${ }^{16}$. The other reason is that during the 7 -day follow-up, confounding factors, such as antibiotics and hospital infection, may interfere the kidney function, thus renal function was assessed and AKI was diagnosed within 48 hours after admission ${ }^{17}$. Because Scr values within 3 months before admission were not recorded in the database, the first measurement of Scr within 24 hours after admitted into ICU was set as the baseline ${ }^{18}$.

Included patients were those: (1) with first admission to ICU during hospital stays; (2) with AKI during 48 hours after admitted to ICU; (3) aged $\geq 18$ years old and $\leq 89$ years old; (4) were followed-up for 4 years by the CareVue system ${ }^{13}$. Patients without any RDW data within 24 hours after admission or missing $>5 \%$ indices were not included in our study. Patients with hospitalization duration longer than 100 days were excluded ${ }^{19}$.

Data extraction. Data of each patient were extracted from the MIMIC-III database by the Structured Query Language, including age, sex, admission type, ethnicity, marriage, status of renal replacement therapy, RDW values, the survival time, days of hospital stay, and days of ICU stay (Additional File 1). If RDW was measured for several times within 24 hours after the ICU entry, data of the first time were used. Comorbidities were defined as per the Implementation of the International Statistical Classification of Disease and Related Health Problems, 10th Revision (ICD-10) coding systems ${ }^{20}$. APS III ${ }^{21}$, the Modified Logistic Organ Dysfunction System $(\text { MLODS })^{22}$, SOFA ${ }^{23}$, the Oxford Acute Severity of Illness Score (OASIS) ${ }^{24}$ and the Systemic Inflammatory Response Syndrome (SIRS) status ${ }^{25}$ were calculated according to the physiological and laboratory parameters in the MIMIC-III database for estimation of prognosis in AKI patients. All methods were carried out in accordance with relevant guidelines to protect the privacy of patients.

Grouping. The data distribution of RDW in all the enrolled subjects was calculated; the lower tertile and the upper tertile were $13.6 \%$ and $15.2 \%$ respectively. We hence divided all of the subjects into three groups based on the tertiles of RDW values 26,27 , namely the low RDW group (RDW $<13.6 \%$ ), the middle RDW group (13.6\% $\leq \mathrm{RDW}<15.2 \%$ ), and the high RDW group (RDW $\geq 15.2 \%$ ). Thereafter, we divided patients into subgroups according to AKI stages which are based on the AKIN guidelines ${ }^{14}$. In specific, AKI stage 1 was defined as AKI with an increase of Scr values $\geq 1.5-1.9$ folds or $\geq 26.5 \mu \mathrm{mol} / \mathrm{L}$, or urine output $<0.5 \mathrm{ml} /(\mathrm{kg} \cdot \mathrm{h})$ for more than 6 hours; AKI stage 2 was defined as AKI with an increase of Scr values $\geq 2.0-2.9$ folds or urine output to $<0.5 \mathrm{ml} /$ (kg.h) for more than 12 hours; AKI stage 3 was defined as AKI with an increase of Scr values $\geq 3.0$ folds or 


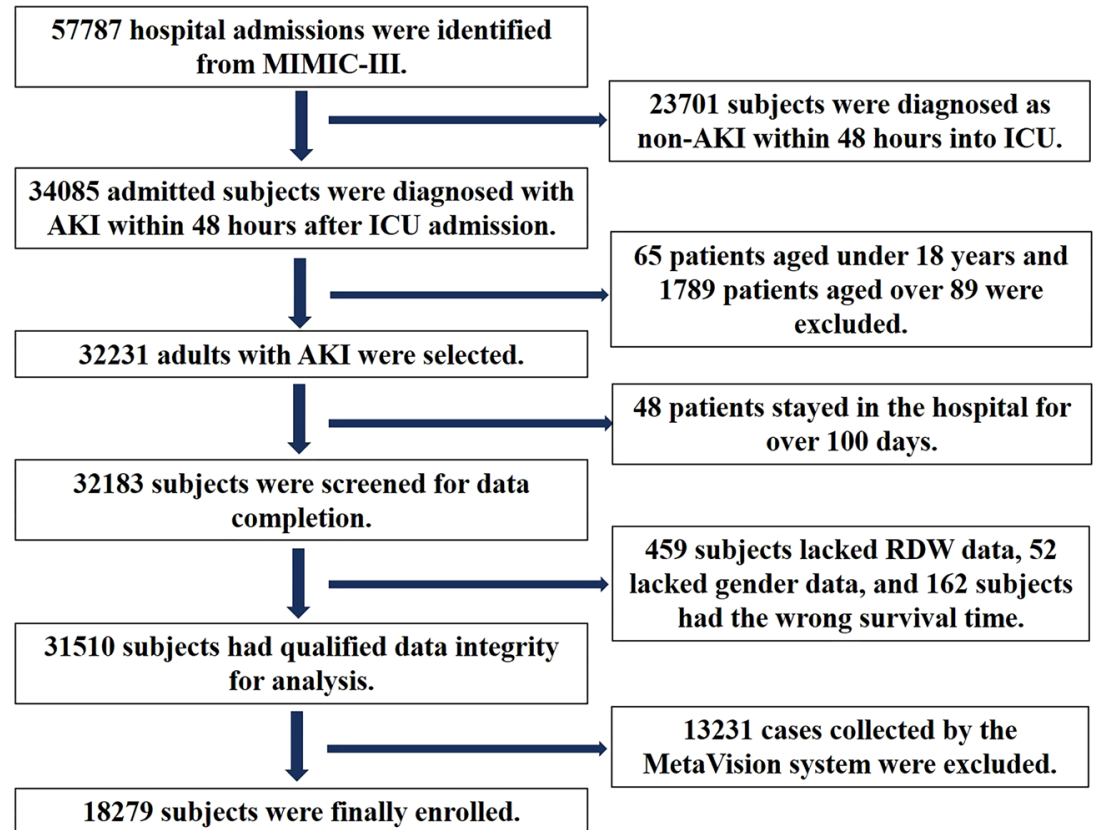

Figure 1. Flowchart of subject screening. Initially, data of 57787 subjects were extracted from the Medical Information Mart for Intensive Care III (MIMIC-III) database. Changes of serum creatinine and urine output within 48 hours after admissions to ICUs were calculated. Then 23701 subjects were excluded as non-acute kidney injury (AKI) patients. Because age of patients over 89 years were marked as 300 or more in the database to protect the privacy, we further excluded 1789 senilities for analysis. After filtering out 65 subjects younger than 18-year-old, 32231 adults were further screened for hospital stays. Hence, 32183 subjects with hospital stay less than 100 days were checked for data integrity, and 459 subjects without red blood cell distribution width (RDW), 52 subjects without sex information and 162 subjects with wrong survival time were excluded. Since the MetaVision system only provided 90-day follow-up data, only 18279 patients with complete follow-up data for 4 years were finally enrolled in our study.

$\geq 354 \mu \mathrm{mol} / \mathrm{L}$, or urine output $<0.3 \mathrm{ml} /(\mathrm{kg} \cdot \mathrm{h})$ for more than 24 hours. Patients with an anuric status $>12$ hours were also classified as AKI stage 3.

Outcomes. The MIMIC-III database provides the follow-up data by two systems, namely the Philips CareVue Clinical Information System (models M2331A and M1215A; Philips Health-care, Andover, MA) for four years and the iMDsoft MetaVision ICU (iMDsoft, Needham, MA) for 90 days. Because we aimed at investigating the long-term prognostic effects of RDW, the four-year outcomes after ICU admission in the CareVue system were used. The all-cause death was used as the end-point in our study. We extracted the death status recorded in CareVue system. Since the MIMIC-III database was connected with social security database, which ensures the integrity of follow-up data, we also replenished all-cause death data in four years for patients without recording in the CareVue system.

Statistics. The trend test of one-way analysis of variance (ANOVA) was used for the analysis of continuous data, and the trend test of Chi-square test was used for categorical data. The linear regression model, the Kaplan-Meier (K-M) curve and the Cox regression model were utilized to analyze the relationship between RDW and survival in AKI patients. The receiver operating characteristic (ROC) curve analysis was performed to compare the area under the ROC curve (AUC), which represented the prognostic efficiency. Statistical analyses were performed with the SPSS 22.0 software (SPSS, IBM, NY, US), GraphPad Prism 7.0 (GraphPad Software, San Diego, US) and the Medcalc 18.5.0 software (MedCalc Software, Ostend, Belgium). Statistical significance was defined as $P<0.05$. Graphs were generated by Medcalc 18.5.0 and GraphPad Prism 7.0 (GraphPad Software, California, US).

\section{Results}

Eighteen thousand two hundred and seventy-nine patients were enrolled. Initially, 57787 subjects were selected from MIMIC-III. After screening, 18279 subjects with mean age of $63.4 \pm 16.2$ years were enrolled (Fig. 1). The demographic data of the enrolled patients were shown in Table 1. Among these patients, $58.3 \%$ were males, and $82.3 \%$ were admitted via the emergency room. As the database was constructed in the United States, a majority of the included patients were Caucasian (69.6\%). According to the KDIGO guideline, patients at AKI stage 2 accounted for 37.2\%, 32.4\% of patients were at AKI stage 3, while the others were at AKI stage 1 . Only $8.8 \%$ of all subjects were treated with renal replacement therapy. Higher severity scale scores represent more severity in critically ill patients with AKI (Table 1). The leading comorbidities were congestive heart 


\begin{tabular}{|c|c|c|c|c|c|}
\hline & \multirow[b]{3}{*}{ All subjects } & \multicolumn{4}{|c|}{ Red blood cell distribution width (\%) } \\
\hline & & \multirow{2}{*}{$\begin{array}{l}<13.6 \\
(\mathrm{~N}=5696)\end{array}$} & \multirow{2}{*}{$\begin{array}{l}13.6-15.2 \\
(\mathrm{~N}=6269)\end{array}$} & \multirow{2}{*}{\begin{tabular}{|l|}
$\geq 15.2$ \\
$(\mathrm{~N}=6314)$
\end{tabular}} & \multirow[b]{2}{*}{$P$} \\
\hline & & & & & \\
\hline Age (years) & $63.4 \pm 16.2$ & $59.6 \pm 17.7$ & $65.3 \pm 15.4$ & $65.0 \pm 14.8$ & $<0.01$ \\
\hline Male & $10661(58.3 \%)$ & $3625(63.6 \%)$ & $3612(57.6 \%)$ & $3424(54.2 \%)$ & $<0.01$ \\
\hline \multicolumn{6}{|l|}{ Admission type } \\
\hline Emergency & $15050(82.3 \%)$ & $4574(80.3 \%)$ & $4964(79.2 \%)$ & $5512(87.3 \%)$ & $<0.01$ \\
\hline Urgent & $641(3.5 \%)$ & $210(3.7 \%)$ & $210(3.3 \%)$ & $221(3.5 \%)$ & \\
\hline Elective & $2588(14.2 \%)$ & $912(16.0 \%)$ & $1095(17.5 \%)$ & $581(9.2 \%)$ & \\
\hline Ethnicity & & & & & $<0.01$ \\
\hline Caucasian & $12714(69.6 \%)$ & $3903(68.5 \%)$ & $4466(71.2 \%)$ & $4345(68.8 \%)$ & \\
\hline Black & $1765(9.7 \%)$ & $334(5.9 \%)$ & $534(8.5 \%)$ & $897(14.2 \%)$ & \\
\hline Asian & $345(1.9 \%)$ & $106(1.9 \%)$ & $121(1.9 \%)$ & $118(1.9 \%)$ & \\
\hline Others & $3455(18.9 \%)$ & $1353(23.8 \%)$ & $1148(18.3 \%)$ & $945(15.1 \%)$ & \\
\hline Marriage & & & & & $<0.01$ \\
\hline Married & $9118(49.9 \%)$ & $2893(50.8 \%)$ & $3188(50.9 \%)$ & $3037(48.1 \%)$ & \\
\hline Single & $4235(23.2 \%)$ & $1376(24.2 \%)$ & $1354(21.6 \%)$ & $1505(23.8 \%)$ & \\
\hline Others & $4926(26.9 \%)$ & $1427(25.1 \%)$ & $1727(27.5 \%)$ & $1772(28.1 \%)$ & \\
\hline AKI stage & & & & & $<0.01$ \\
\hline 1 & $5557(30.4 \%)$ & $1751(30.8 \%)$ & $1834(29.3 \%)$ & $1970(31.2 \%)$ & \\
\hline 2 & $6798(37.2 \%)$ & $2233(39.2 \%)$ & $2529(40.3 \%)$ & $2036(32.2 \%)$ & \\
\hline 3 & $5924(32.4 \%)$ & $1710(30.0 \%)$ & $1906(30.4 \%)$ & $2308(36.6 \%)$ & \\
\hline \multicolumn{6}{|l|}{ Renal replacement therapy } \\
\hline Yes & $1616(8.8 \%)$ & $108(1.9 \%)$ & $334(5.3 \%)$ & $1174(18.6 \%)$ & $<0.01$ \\
\hline No & $16663(92.4 \%)$ & $5588(98.1 \%)$ & $5935(94.7 \%)$ & $5140(81.4 \%)$ & \\
\hline \multicolumn{6}{|l|}{ Severity scale } \\
\hline APS III & $44.71 \pm 19.71$ & $37.87 \pm 17.07$ & $43.51 \pm 18.74$ & $52.07 \pm 20.38$ & $<0.01$ \\
\hline MLODS & $2.89 \pm 2.41$ & $2.22 \pm 2.18$ & $2.83 \pm 2.37$ & $3.57 \pm 2.46$ & $<0.01$ \\
\hline SOFA & $4.52 \pm 3.09$ & $3.59 \pm 2.61$ & $4.39 \pm 2.92$ & $5.51 \pm 3.35$ & $<0.01$ \\
\hline OASIS & $32.08 \pm 8.01$ & $30.79 \pm 8.47$ & $32.23 \pm 8.62$ & $33.09 \pm 9.13$ & $<0.01$ \\
\hline SIRS & $2.85 \pm 1.00$ & $2.84 \pm 1.02$ & $2.88 \pm 0.99$ & $2.82 \pm 0.98$ & $<0.01$ \\
\hline \multicolumn{6}{|l|}{ Comorbidity } \\
\hline Chronic pulmonary disease & $3389(18.5 \%)$ & $745(13.1 \%)$ & $1265(20.2 \%)$ & $1379(21.8 \%)$ & $<0.01$ \\
\hline Congestive heart failure & $5831(31.9 \%)$ & $1001(17.6 \%)$ & $2069(33.0 \%)$ & $2761(43.7 \%)$ & $<0.01$ \\
\hline Liver disease & $1363(7.5 \%)$ & $109(1.9 \%)$ & $338(5.4 \%)$ & $916(14.5 \%)$ & $<0.01$ \\
\hline Metastatic cancer & $924(5.1 \%)$ & $125(2.2 \%)$ & $269(4.3 \%)$ & $530(8.4 \%)$ & $<0.01$ \\
\hline Renal failure & $2898(15.9 \%)$ & $216(3.8 \%)$ & $738(11.8 \%)$ & $1944(30.8 \%)$ & $<0.01$ \\
\hline Time in hospital (days) & $11.3 \pm 10.8$ & $9.6 \pm 9.3$ & $11.1 \pm 10.4$ & $12.9 \pm 12.2$ & $<0.01$ \\
\hline Time in ICU (days) & $5.1 \pm 7.2$ & $4.5 \pm 6.5$ & $5.1 \pm 7.3$ & $5.6 \pm 7.6$ & $<0.01$ \\
\hline Death & $7973(43.6 \%)$ & $1314(23.1 \%)$ & $2507(40 \%)$ & $4152(65.8 \%)$ & $<0.01$ \\
\hline
\end{tabular}

Table 1. Demographic data of study subjects. Note: AKI, acute kidney injury; APS III, acute physiology and chronic health evaluation III; MLODS, modified logistic organ dysfunction system; SOFA, sequential organ failure assessment; OASIS, oxford acute severity of illness score; SIRS, systemic inflammatory response syndrome; ICU, intensive care unit.

failure and chronic pulmonary disease, which accounted for $31.9 \%$ and $18.5 \%$, respectively, and $15.9 \%$ of the patients were complicated with renal failure. The median hospital stay of all patients was 7.9 days and the median ICU stay was 2.7 days. The all-cause death rate for all subjects was $43.6 \%$.

RDW values were negatively correlated with survival time of critically ill patients with AKI. Demographic data were compared, and all parameters, including gender, age, admission type, marriage, ethnicity, AKI stage, traditional severity scores, comorbidities, ICU stay and hospital stay, have shown differences among the three RDW groups (all $P<0.01$, Table 1). In all subjects, the death rate was increased from low to high RDW groups (23.1\% for the low RDW group, $40 \%$ for the middle RDW group, $65.8 \%$ for the high RDW group, Table 1 and Fig. 2A), while the survival time was decreased from low to high RDW groups (Fig. 2B). Similar trends in death rate and survival time were found at each AKI stage (Fig. 2A,B). In the linear regression model, survival time was negatively correlated to RDW values $(r=0.35, P<0.01)$.

RDW was an independent risk factor of 4-year all-cause mortality in critically ill patients with

AKI. By the K-M analysis, higher RDW values were associated with lower survival rate and shorter survival 


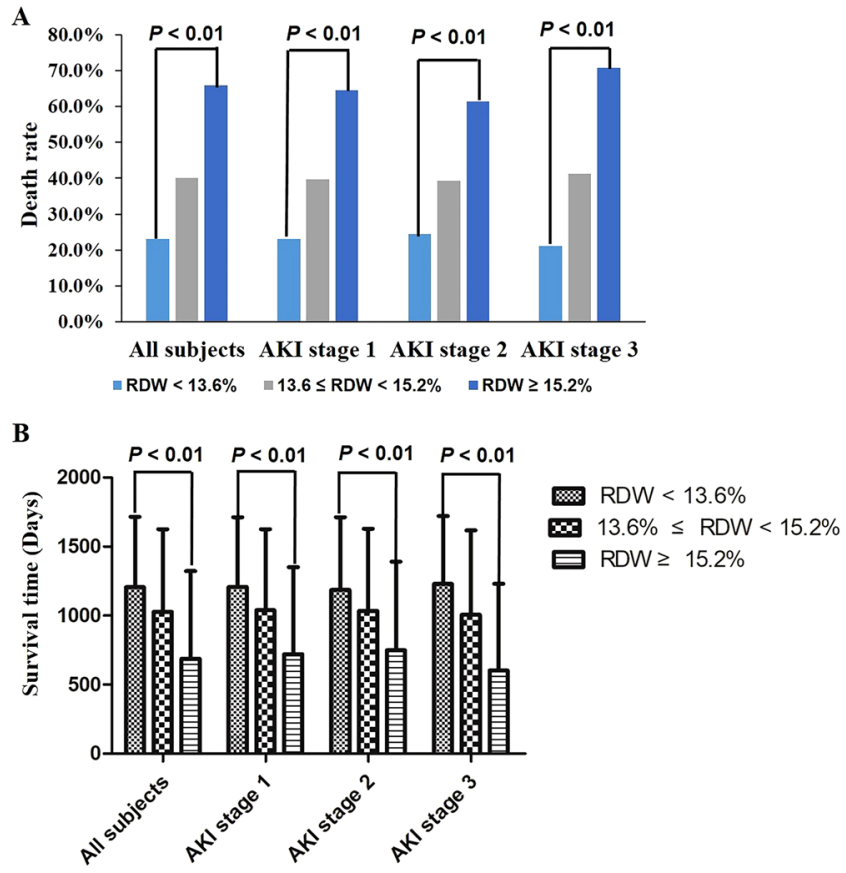

Figure 2. Relationship between red blood cell distribution width (RDW) and all-cause death in critically ill patients with acute kidney injury (AKI). AKI patients were divided into the low (RDW $<13.6 \%)$, middle $(13.6 \% \leq \mathrm{RDW}<15.2 \%)$ and high $(\mathrm{RDW} \geq 15.2 \%) \mathrm{RDW}$ groups according to the first RDW measurement. Death rates increased from the low RDW group to the high RDW group for all patients $(23.10 \%, 40.00 \%$ and $65.80 \%$ for the low, the middle and the high RDW groups respectively, $P<0.01, \mathbf{A}$ ). The same relationship was also found at AKI stage 1 (23.20\%, 39.70\% and 64.50\% for the low, middle and high RDW groups respectively, $P<0.01)$, stage $2(24.50 \%, 39.30 \%$ and $61.30 \%$ for the low, middle and high RDW groups respectively, $P<0.01)$ and stage $3(21.10 \%, 41.20 \%$ and $70.80 \%$ for the low, middle and high RDW groups respectively, $P<0.01)$. With the RDW increasing, survival time decreased from $1205.7 \pm 509.5$ days to $687.0 \pm 635.8$ days $(P<0.01, B)$. In subgroup analysis, survival time of each RDW groups were also compared at each AKI stage separately, and AKI patients of low RDW group had the longest survival time at all stages $(P<0.01, \mathbf{B})$.

time $(P<0.01$ by logrank test for trend, Fig. $3 \mathrm{~A})$. We also conducted subgroup analysis of AKI stage 1 , stage 2 and stage 3 groups respectively by the K-M curve, while patients with higher RDW values also showed shorter survival time and higher death rate at each stage $(P<0.01$ by logrank test for trend, Fig. $3 \mathrm{~B}-\mathrm{D})$. Then the Cox regression models were performed for the association between RDW and all-cause mortality independently as well as adjusted by other severity scale scores. RDW was independently related to the all-cause mortality of AKI patients both in unadjusted and adjusted Cox regression models $(P<0.01$, Table 2$)$. RDW was also shown as an independent risk factor for all-cause mortality at each AKI stage. In Cox regression models, RDW remained a risk factor of mortality of AKI patients before and after adjustment (Table 3).

Effects of RDW on 4-year all-cause mortality were not influenced by the history of renal failure in critically ill patients with AKI. Baseline renal function may have an impact on the incidence and mortality of AKI. Hence, we did the survival analysis of RDW on patients without renal failure. The K-M curves indicated that high RDW values were associated with higher all-cause mortality and shorter survival time in all subjects as well as patients at different AKI stages $(P<0.01$, Fig. 4$)$. Thus, regardless of the baseline renal function, RDW was an independent risk factor for long-term mortality in AKI patients.

RDW predicted long-term prognosis better than conventional severity scales. The ROC analysis was performed to evaluate the long-term prognostic value of RDW compared with conventional severity scale scores, including APS III, MLODS, OASIS, SIRS and SOFA. The AUC of RDW for all patients was 0.718 (95\% CI, 0.712-0.725), which was significantly higher than other severity scale scores $(P<0.01$, Fig. 5A and Table 4). For patients at AKI stage 1, RDW with AUC of 0.713 (95\% CI, 0.701-0.725) had a better prognostic value than conventional severity scales $(P<0.01$, Fig. 5B and Table 4$)$. However, for patients with AKI stage 2 and 3, AUC of RDW showed no significant difference from APS III $(P=0.297$ for AKI stage $2, P=0.781$ for AKI stage 3, Fig. 5C,D and Table 4). When RDW was combined with each severity score, AUCs were significantly increased after combination as compared with APS III, MLODS, OASIS, SOFA or RDW alone, respectively (all $P<0.01$, Figure S1 and Table 4). However, no difference of AUC was found after combination of RDW with SIRS ( $P=0.080$, Figure S1 and Table 4$)$. Taken together, RDW better predicts long-term prognosis of critically ill patients with AKI than traditional severity scoring systems. RDW may also improve the prognostic efficiency of APS III. 
A

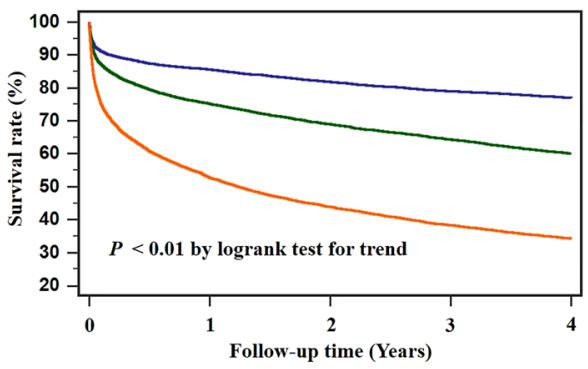

Number of patients at risk

$\begin{array}{llllll}\text { Low RDW } & 5696 & 4877 & 4661 & 4500 & 4382 \\ \text { Middle RDW } & 6269 & 4714 & 4324 & 4033 & 3762 \\ \text { High RDW } & 6314 & 3330 & 2773 & 2420 & 2162\end{array}$

K-M curve of all AKI patients

C

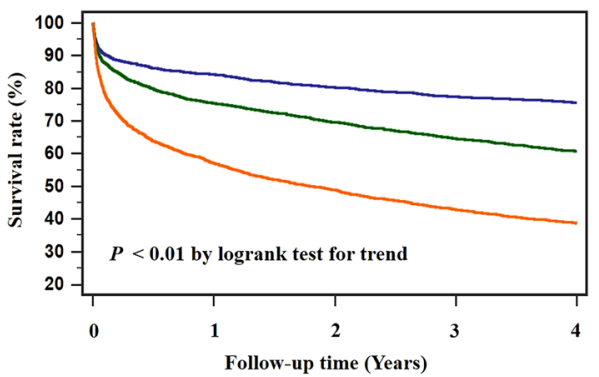

Number of patients at risk

$\begin{array}{lccccc}\text { Low RDW } & 2233 & 1882 & 1793 & 1728 & 1687 \\ \text { Middle RDW } & 2529 & 1908 & 1761 & 1635 & 1535 \\ \text { High RDW } & 2036 & 1162 & 997 & 874 & 787\end{array}$

High RDW 2036

K-M curve of AKI stage 2 patients

B

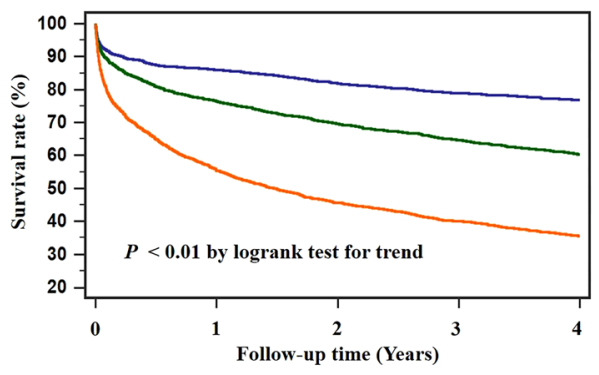

Number of patients at risk

$\begin{array}{llllll}\text { Low RDW } & 1753 & 1507 & 1435 & 1383 & 1346\end{array}$

Middle RDW 1834

High RDW 1970

$1402 \quad 1277$

1185

$1093 \quad 901 \quad 789$

K-M curve of AKI stage 1 patients

D

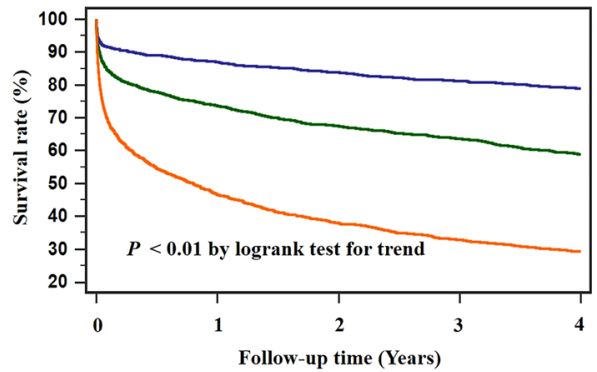

Number of patients at risk

$\begin{array}{llllll}\text { Low RDW } & 1710 & 1488 & 1433 & 1389 & 1349\end{array}$

Middle RDW 1906

High RDW 2308

Figure 3. Kaplan-Meier (K-M) survival curves for 4-year all-cause mortalities of critically ill patients with acute kidney injury (AKI). The risk of 4-year overall mortality of each red blood cell distribution width (RDW) group in all participants was shown (A). Patients with $\mathrm{RDW} \geq 15.2 \%$ had the highest all-cause mortality. In subgroup analysis, the same changes of the Kaplan-Meier curves were observed at AKI stage 1 (B), stage 2 (C) and stage 3 (D).

\begin{tabular}{|c|c|c|c|c|c|c|c|c|}
\hline & \multicolumn{2}{|c|}{ All subjects $(\mathrm{N}=18279)$} & \multicolumn{2}{|c|}{ AKI stage $1(\mathrm{~N}=5557)$} & \multicolumn{2}{|c|}{ AKI stage $2(\mathrm{~N}=6798)$} & \multicolumn{2}{|c|}{ AKI stage $3(\mathrm{~N}=5924)$} \\
\hline & HR $(95 \% \mathrm{CI})$ & $P$ & HR $(95 \% \mathrm{CI})$ & $P$ & HR $(95 \% \mathrm{CI})$ & $P$ & HR $(95 \% \mathrm{CI})$ & $P$ \\
\hline Unadjusted RDW & $1.219(1.211,1.228)$ & $<0.01$ & $1.214(1.197,1.230)$ & $<0.01$ & $1.202(1.185,1.218)$ & $<0.01$ & $1.231(1.217,1.245)$ & $<0.01$ \\
\hline \multicolumn{9}{|c|}{ RDW adjusted for } \\
\hline APS III & $1.173(1.164,1.182)$ & $<0.01$ & $1.185(1.168,1.202)$ & $<0.01$ & $1.172(1.155,1.189)$ & $<0.01$ & $1.159(1.144,1.174)$ & $<0.01$ \\
\hline MLODS & $1.194(1.185,1.203)$ & $<0.01$ & $1.193(1.176,1.210)$ & $<0.01$ & $1.190(1.174,1.207)$ & $<0.01$ & $1.185(1.171,1.200)$ & $<0.01$ \\
\hline SOFA & $1.187(1.178,1.196)$ & $<0.01$ & $1.189(1.172,1.207)$ & $<0.01$ & $1.189(1.173,1.206)$ & $<0.01$ & $1.173(1.158,1.187)$ & $<0.01$ \\
\hline OASIS & $1.213(1.204,1.222)$ & $<0.01$ & $1.225(1.208,1.242)$ & $<0.01$ & $1.199(1.182,1.216)$ & $<0.01$ & $1.202(1.188,1.217)$ & $<0.01$ \\
\hline SIRS & $1.222(1.211,1.229)$ & $<0.01$ & $1.215(1.198,1.232)$ & $<0.01$ & $1.202(1.186,1.219)$ & $<0.01$ & $1.228(1.214,1.242)$ & $<0.01$ \\
\hline
\end{tabular}

Table 2. Relationship between RDW and all-cause mortality in Cox model before and after adjustment. Note: AKI, acute kidney injury; HR, hazard ratio; CI, confidence interval; SE, standard error; RDW, red blood cell distribution width; APS III, acute physiology and chronic health evaluation III; MLODS, modified logistic organ dysfunction system; SOFA, sequential organ failure assessment; OASIS, oxford acute severity of illness score; SIRS, systemic inflammatory response syndrome.

\section{Discussion}

Clinically, RDW is a common parameter for evaluation of anemia and inflammation. In recent years, much attention has been paid to the relationship between renal function and RDW. In this study, we explored the association between RDW and long-term outcomes of 18279 critically ill patients with AKI. Higher levels of RDW predicted shorter survival time and higher long-term death rate. Our findings suggest that RDW might be a potential predictor of all-cause mortality of critically ill patients with AKI.

First, we explored the association between RDW and survival time. We demonstrated that higher RDW predicted worse prognosis of AKI patients. Our results were consistent with previous studies on short-term 


\begin{tabular}{|c|c|c|c|c|c|c|c|c|}
\hline & \multicolumn{2}{|c|}{ All subjects $(\mathrm{N}=15381)$} & \multicolumn{2}{|c|}{ AKI stage $1(\mathrm{~N}=4520)$} & \multicolumn{2}{|c|}{ AKI stage $2(\mathrm{~N}=6174)$} & \multicolumn{2}{|c|}{ AKI stage $3(\mathrm{~N}=4687)$} \\
\hline & HR $(95 \%$ CI) & $P$ & HR $(95 \%$ CI $)$ & $P$ & HR $(95 \% \mathrm{CI})$ & $P$ & HR $(95 \% \mathrm{CI})$ & $P$ \\
\hline Unadjusted RDW & $1.221(1.210,1.231)$ & $<0.01$ & $1.212(1.192,1.233)$ & $<0.01$ & $1.206(1.188,1.224)$ & $<0.01$ & $1.234(1.218,1.250)$ & $<0.01$ \\
\hline \multicolumn{9}{|l|}{ RDW adjusted for } \\
\hline APS III & $1.177(1.166,1.188)$ & $<0.01$ & $1.189(1.168,1.211)$ & $<0.01$ & $1.177(1.159,1.195)$ & $<0.01$ & $1.160(1.143,1.178)$ & $<0.01$ \\
\hline MLODS & $1.202(1.191,1.213)$ & $<0.01$ & $1.205(1.184,1.226)$ & $<0.01$ & $1.197(1.179,1.216)$ & $<0.01$ & $1.190(1.173,1.207)$ & $<0.01$ \\
\hline SOFA & $1.191(1.181,1.202)$ & $<0.01$ & $1.199(1.178,1.220)$ & $<0.01$ & $1.194(1.176,1.212)$ & $<0.01$ & $1.171(1.154,1.188)$ & $<0.01$ \\
\hline OASIS & $1.212(1.202,1.223)$ & $<0.01$ & $1.220(1.200,1.242)$ & $<0.01$ & $1.201(1.183,1.219)$ & $<0.01$ & $1.205(1.188,1.222)$ & $<0.01$ \\
\hline SIRS & $1.220(1.210,1.230)$ & $<0.01$ & $1.214(1.193,1.234)$ & $<0.01$ & $1.206(1.188,1.224)$ & $<0.01$ & $1.230(1.213,1.246)$ & $<0.01$ \\
\hline
\end{tabular}

Table 3. Relationship between RDW and all-cause mortality of patients without chronic kidney disease in Cox model before and after adjustment. Note: AKI, acute kidney injury; HR, hazard ratio; CI, confidence interval; SE, standard error; RDW, red blood cell distribution width; APS III, acute physiology and chronic health evaluation III; MLODS, modified logistic organ dysfunction system; SOFA, sequential organ failure assessment; OASIS, oxford acute severity of illness score; SIRS, systemic inflammatory response syndrome.

A
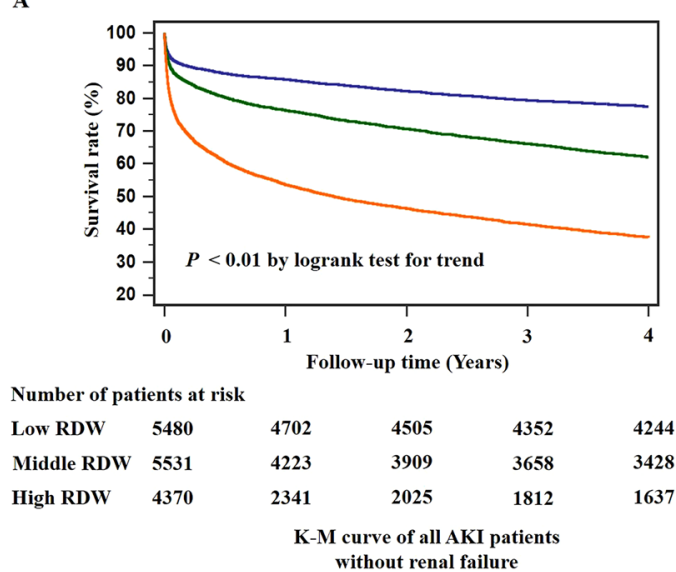

C

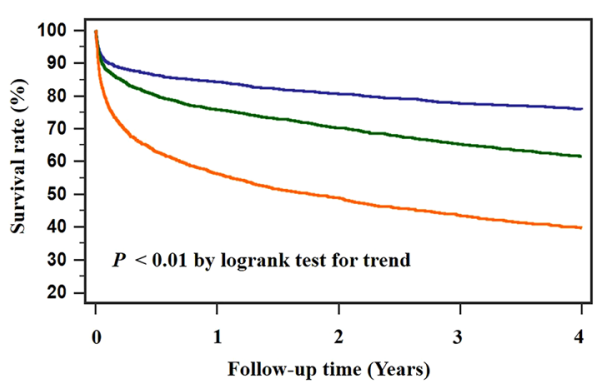

Number of patients at risk

$\begin{array}{llllll}\text { Low RDW } & 2172 & 1833 & 1752 & 1689 & 1648\end{array}$

$\begin{array}{lllllll}\text { Middle RDW } & 2310 & 1753 & 1623 & 1509 & 1422\end{array}$

High RDW 1692
$952 \quad 828 \quad 737$

K-M curve of AKI stage 2 patients without renal failure
B

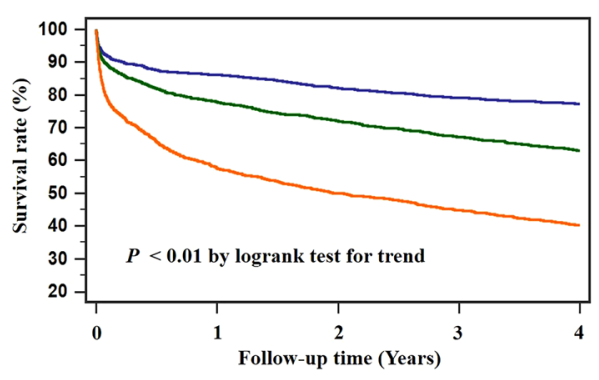

Number of patients at risk

$\begin{array}{llllll}\text { Low RDW } & 1673 & 1441 & 1373 & 1323 & 1293\end{array}$

Middle RDW 1582

High RDW 1265

1231

1140

1063

567

K-M curve of AKI stage 1 patients without renal failure

D

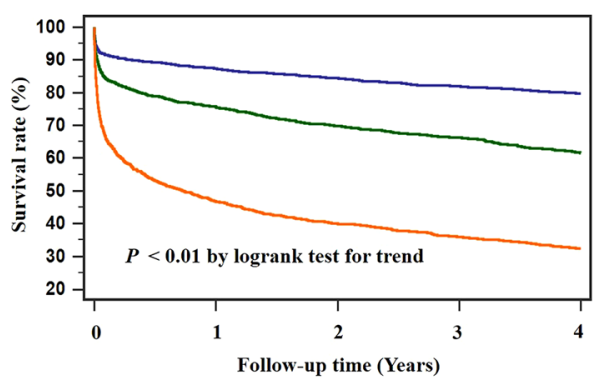

Number of patients at risk

$\begin{array}{llllll}\text { Low RDW } & 1635 & 1428 & 1380 & 1340 & 1303\end{array}$

Middle RDW 1639

High RDW 1413 $\begin{array}{cccc}1428 & 1380 & 1340 & 1303 \\ 1239 & 1146 & 1086 & 1010 \\ 661 & 565 & 508 & 458\end{array}$

K-M curve of AKI stage 3 patients without renal failure

- $\mathrm{RDW}<13.6 \%-13.6 \% \leq \mathrm{RDW}<15.2 \%-\mathrm{RDW} \geq 15.2 \%$

Figure 4. Kaplan-Meier (K-M) survival curves for 4-year overall mortalities of critically ill patients with acute kidney injury (AKI) without comorbidity of renal failure. To eliminate the influences of baseline renal function, we did the Kaplan-Meier analysis for each red blood cell distribution width (RDW) groups in patients without renal failure. The survival rate of the low RDW group was significantly higher than the middle and high RDW groups $(P<0.01)$ for all patients in the 4-year follow-up $(\mathbf{A})$. Higher risk of mortality was also shown in the high RDW group at AKI stage $1(P<0.01, \mathbf{B}), 2(P<0.01, \mathbf{C})$ and $3(P<0.01, \mathbf{D})$ respectively.

outcomes, which mainly focused on how RDW was related with survival in AKI patients after percutaneous transluminal coronary intervention or with other coronary diseases, especially contrast-induced $\mathrm{AKI}^{8,28,29}$. Survivors of AKI had a lower RDW value. Similar findings about the association between low RDW and high survival rate were also reported in sepsis-induced ${ }^{30}$ and extracorporeal membrane oxygenation treated patients with 
A

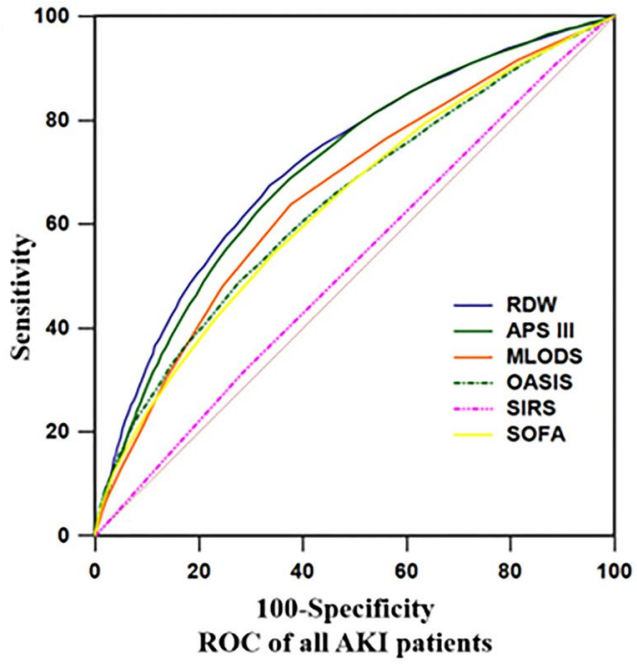

C

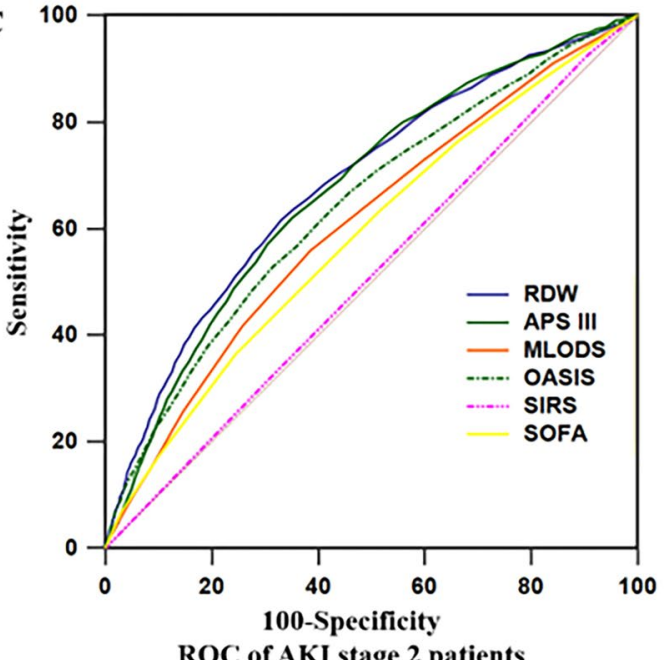

B

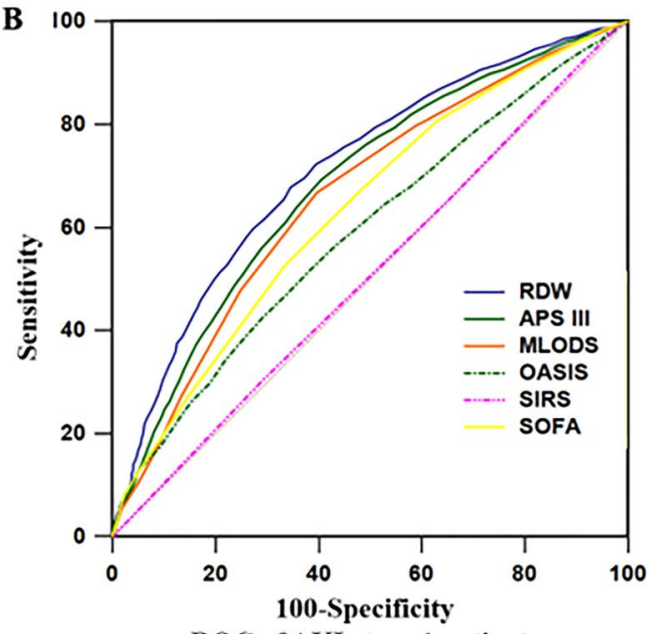

ROC of AKI stage 1 patients

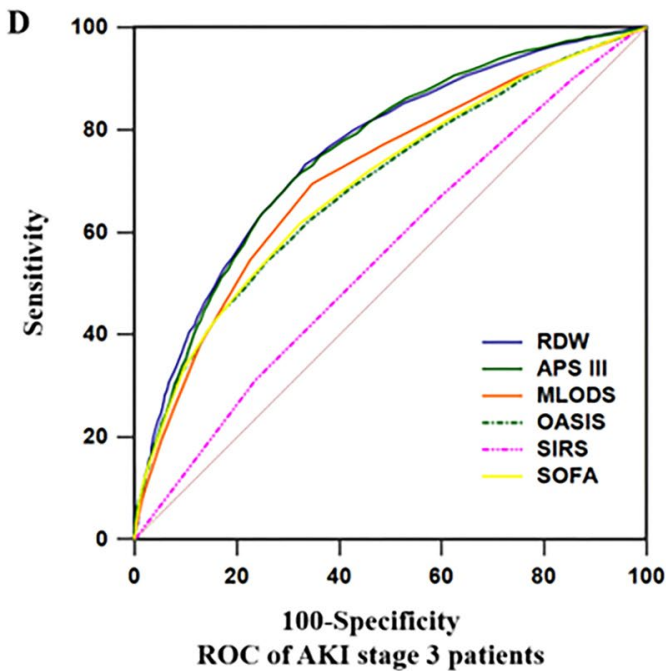

Figure 5. Receiver operating curve (ROC) analyses of predictors of acute kidney injury (AKI) and mortality in intensive care units (ICU) patients. The area under ROC curve (AUC) of red blood cell distribution width (RDW) were compared with Acute Physiology Score III (APS III), the Modified Logistic Organ Dysfunction System (MLODS), the Sequential Organ Failure Assessment (SOFA), the Oxford Acute Severity of Illness Score (OASIS) and the Systemic Inflammatory Response Syndrome (SIRS). RDW values of all subjects (AUC $=0.718$, A) and AKI stage 1 subjects ( $\mathrm{AUC}=0.713, \mathbf{B})$ were significantly higher than other severity scale scores $(P<0.01)$. However, at AKI stages 2 and 3, the AUCs of RDW (AUC $=0.684$ for stage 2 in $C$ and AUC $=0.757$ for stage 3 in D) showed no difference in comparison with APS III ( $P=0.297$ for stage 2 and $P=0.781$ for stage 3 ).

AKI. Different from previous studies, however, we emphasized that the baseline RDW values also determined the long-term death rate and survival time in multiple-cause AKI.

To adjust for the confounding of baseline renal function, we further examined the relationship between RDW and long-term all-cause mortalities of AKI patients without comorbidity of renal failure. Elevation of blood RDW levels has been used to predict mortalities in chronic kidney disease (CKD) patients both with and without hemodialysis ${ }^{31,32}$. For patients with normal baseline renal function, RDW was positively correlated to 4 -year survival rates, which was consistent with Odutayo's results ${ }^{33}$. Hence RDW may serve as an independent factor of long-term outcomes of critically ill patients with AKI.

We also compared the prognostic value of RDW with traditional severity scores. Oh's team analyzed the data of 470 renal replacement therapy-treated patients with AKI and found that SOFA with the AUC of 0.694 predicted more accurately than RDW with the AUC of $0.586^{34}$. Mizuno and colleagues showed the potential predictive ability of RDW combined with the Mehran risk score but not RDW only for contrast-induced AKI in myocardial infarction patients ${ }^{35}$. Acute physiology and chronic health evaluation II scores were also confirmed to be better than RDW in predicting in-hospital mortality as well as 2 -year mortality ${ }^{8}$. Inconsistent with previous studies, however, RDW showed a better predictive value of four-year mortality than commonly used severity scoring systems in our study. This inconsistency might be due to differences in sample size and selection of subjects. On one hand, previous studies usually enrolled no more than 1000 subjects, which is far less than the sample size in 


\begin{tabular}{|c|c|c|c|c|c|c|c|c|}
\hline & \multicolumn{2}{|c|}{ All subjects $(\mathrm{N}=18279)$} & \multicolumn{2}{|c|}{ AKI stage $1(\mathrm{~N}=5557)$} & \multicolumn{2}{|c|}{ AKI stage $2(\mathrm{~N}=6798)$} & \multicolumn{2}{|c|}{ AKI stage $3(\mathrm{~N}=5924)$} \\
\hline & $\operatorname{AUC}(95 \% \mathrm{CI})$ & $P$ & $\operatorname{AUC}(95 \% \mathrm{CI})$ & $P$ & $\operatorname{AUC}(95 \% \mathrm{CI})$ & $P$ & $\operatorname{AUC}(95 \% \mathrm{CI})$ & $P$ \\
\hline RDW & $0.718(0.712-0.725)$ & & $0.713(0.701-0.725)$ & & $0.684(0.673-0.695)$ & & $0.757(0.746-0.768)$ & \\
\hline APS III & $0.706(0.700-0.713)$ & $<0.01$ & $0.682(0.670-0.695)$ & $<0.01$ & $0.675(0.664-0.686)$ & 0.297 & $0.755(0.744-0.766)$ & 0.781 \\
\hline RDW + APS III & $0.758(0.752-0.764)$ & $<0.01$ & $0.741(0.729-0.752)$ & $<0.01$ & $0.725(0.714-0.735)$ & $<0.01$ & $0.802(0.792-0.812)$ & $<0.01$ \\
\hline MLODS & $0.659(0.652-0.666)$ & $<0.01$ & $0.660(0.647-0.672)$ & $<0.01$ & $0.608(0.596-0.619)$ & $<0.01$ & $0.709(0.698-0.721)$ & $<0.01$ \\
\hline RDW + MLODS & $0.741(0.735-0.748)$ & $<0.01$ & $0.734(0.722-0.745)$ & $<0.01$ & $0.700(0.689-0.711)$ & $<0.01$ & $0.787(0.776-0.797)$ & $<0.01$ \\
\hline SOFA & $0.638(0.631-0.645)$ & $<0.01$ & $0.633(0.620-0.646)$ & $<0.01$ & $0.586(0.574-0.597)$ & $<0.01$ & $0.695(0.683-0.707)$ & $<0.01$ \\
\hline RDW + SOFA & $0.732(0.726-0.739)$ & $<0.01$ & $0.724(0.712-0.736)$ & $<0.01$ & $0.689(0.678-0.700)$ & 0.115 & $0.780(0.769-0.791)$ & $<0.01$ \\
\hline OASIS & $0.642(0.635-0.649)$ & $<0.01$ & $0.590(0.577-0.603)$ & $<0.01$ & $0.643(0.631-0.654)$ & $<0.01$ & $0.691(0.679-0.702)$ & $<0.01$ \\
\hline RDW + OASIS & $0.750(0.743-0.756)$ & $<0.01$ & $0.726(0.714-0.738)$ & $<0.01$ & $0.727(0.717-0.738)$ & $<0.01$ & $0.792(0.781-0.802)$ & $<0.01$ \\
\hline SIRS & $0.522(0.514-0.529)$ & $<0.01$ & $0.506(0.493-0.519)$ & $<0.01$ & $0.510(0.498-0.522)$ & $<0.01$ & $0.554(0.542-0.567)$ & $<0.01$ \\
\hline RDW + SIRS & $0.720(0.713-0.726)$ & 0.080 & $0.713(0.701-0.725)$ & 0.964 & $0.685(0.674-0.696)$ & 0.402 & $0.760(0.749-0.771)$ & 0.018 \\
\hline
\end{tabular}

Table 4. Area under receiver operating curve of RDW and severity scales at different AKI stages. Note: AKI, acute kidney injury; AUC, area under curve; CI, confidence interval; SE, standard error; RDW, red blood cell distribution width; APS III, acute physiology and chronic health evaluation III; MLODS, modified logistic organ dysfunction system; SOFA, sequential organ failure assessment; OASIS, Oxford acute severity of illness score; SIRS, systemic inflammatory response syndrome.

our study. A large sample could make the results more precise, stable and reliable ${ }^{36}$. On the other hand, subjects in the present study were critically ill patients with AKI caused by various reasons. Heterogeneous causes of AKI might also influence the prognostic efficiency of RDW. Likewise, different factors that may influence short-term and long-term outcomes should be addressed as well ${ }^{37}$. Although traditional severity scales are used mainly to evaluate disease severity and short-term prognosis of acute diseases, studies have proved their usefulness in the long-term prognosis prediction. For example, Pekkarinen's study indicated that SOFA was associated with 1-year outcome and healthcare costs of patients with cardiac arrest ${ }^{38}$. Hagen's team found that SIRS could predict poor long-term functional outcome after intracerebral hemorrhage ${ }^{39}$. In line with these findings, we also showed that either RDW or severity scores could be used to predict the long-term prognosis of AKI patients; RDW appeared to be even more efficient.

Although the predictive value of RDW on all-cause mortalities has been revealed, the mechanism remains unknown. Both inflammation ${ }^{40}$ and oxidative stress ${ }^{41}$ may play crucial roles. When inflammation occurs, iron metabolism and bone marrow function are inhibited, and the proliferation and maturation of erythrocytes are inhibited ${ }^{40}$, leading to an increase of RDW values. Meanwhile, the severity of AKI is related to systematic and intrarenal inflammation ${ }^{42}$. SIRS mainly represents an inflammation status of whole body, and our results revealed that SIRS values were increased in the high RDW group. Therefore, RDW could predict the prognosis of AKI partially by reflecting the levels of inflammation. AKI patients in ICU are often complicated with activation of oxidative stress including disturbed metabolism, sepsis, and hemodynamic dysregulations ${ }^{43}$. Oxidative stress plays key roles in the erythroid cell cycle, differentiation and maturation, and RDW has been found associated with various oxidative stress biomarkers, such as serum malondialdehyde and tumor necrosis factor-alpha, in critically ill patients ${ }^{44}$. Usually, the multi-organ dysfunction is accompanied with severe oxidative stress. Hence, elevations of MLODS and SOFA were shown in the high RDW groups.

The large sample size is the advantage of our study. The complete follow-up data make it possible to investigate the long-term prognosis, which is difficult to be observed in cohort studies. However, limitations should be acknowledged. First, as mentioned in the Subjects and methods section, the AKIN criteria instead of the KDIGO criteria was used due to the intrinsic drawback of the MIMIC-III database. Second, some important information was not recorded in the MIMIC-III database, including Scr during the previous 3 months and the past medical history of end stage renal disease (ESRD), and hence estimation of previousrenal function and exclusion of patients with ESRD were impossible. Third, given the missing items of past medical histories in the MIMIC-III database, patients with hematological diseases or renal anemia were difficult to distinguish and select. Hence, effects of hematological diseases on the prognostic values of RDW were not evaluated. Besides, the missing data of some other RDW-influencing pre-existing conditions, such as serious infections, autoimmune diseases and so forth, also made stratified analyses impossible. Fourth, because the major adverse events after discharge were not followed or recorded, the predictive value of RDW on cardiovascular and cerebrovascular events was not evaluated as well.

Further improvement in the MIMIC-III database may make up for the above-mentioned limitations. Regular follows-up for up to 3 to 6 years are necessary. Detailed past medical history, medication history and the exact cause of death should be recorded, which are helpful to explore the prognostic values of RDW on important complications in critically ill patients with AKI.

\section{Conclusion}

Based on the clinical data of 18279 AKI patients in MIMIC-III database, a negative correlation was found between RDW and all-cause mortality. RDW proved to be a predictive parameter of long-term prognosis of critically ill patients with AKI. 


\section{Data availability}

All data and material were available at https://mimic.mit.edu/.

Received: 14 January 2019; Accepted: 28 February 2020;

Published online: 12 March 2020

\section{References}

1. Darmon, M. et al. Diagnostic work-up and specific causes of acute kidney injury. Intensive Care Med. 43, 829-840, https://doi. org/10.1007/s00134-017-4799-8 (2017).

2. Hoste, E. A. et al. Epidemiology of acute kidney injury in critically ill patients: the multinational AKI-EPI study. Intensive Care Med. 41, 1411-1423, https://doi.org/10.1007/s00134-015-3934-7 (2015).

3. Mehta, R. L. et al. Sepsis as a cause and consequence of acute kidney injury: Program to Improve Care in Acute Renal Disease. Intensive Care Med. 37, 241-248, https://doi.org/10.1007/s00134-010-2089-9 (2011).

4. da Rocha, E. P. et al. Urinary Neutrophil Gelatinase-Associated Lipocalin Is Excellent Predictor of Acute Kidney Injury in Septic Elderly Patients. Aging Dis. 9, 182-191, https://doi.org/10.14336/ad.2017.0307 (2018).

5. Ivey-Miranda, J. B. et al. Right ventricular longitudinal strain predicts acute kidney injury and short-term prognosis in patients with right ventricular myocardial infarction. Int J Cardiovasc Imaging, 107-116, https://doi.org/10.1007/s10554-018-1447-5 (2018).

6. Ivan, M. V. et al. New Molecular and Epigenetic Expressions as Novel Biomarkers in Critically Ill Polytrauma Patients with Acute Kidney Injury (AKI). Clin. Lab. 64, 663-668, https://doi.org/10.7754/Clin.Lab.2018.171226 (2018).

7. Coca, S. G., Singanamala, S. \& Parikh, C. R. Chronic kidney disease after acute kidney injury: a systematic review and meta-analysis. Kidney Int. 81, 442-448, https://doi.org/10.1038/ki.2011.379 (2012).

8. Hu, Y., Liu, H., Fu, S., Wan, J. \& Li, X. Red Blood Cell Distribution Width is an Independent Predictor of AKI and Mortality in Patients in the Coronary Care Unit. Kidney Blood Press. Res. 42, 1193-1204, https://doi.org/10.1159/000485866 (2017).

9. Han, Y. Q. et al. Red blood cell distribution width predicts long-term outcomes in sepsis patients admitted to the intensive care unit. Clin. Chim. Acta 487, 112-116, https://doi.org/10.1016/j.cca.2018.09.019 (2018).

10. Goyal, H., Awad, H. \& Hu, Z. D. Prognostic value of admission red blood cell distribution width in acute pancreatitis: a systematic review. Ann. Transl. Med. 5, 342, https://doi.org/10.21037/atm.2017.06.61 (2017).

11. Wang, B., Lu, H., Gong, Y., Ying, B. \& Cheng, B. The Association between Red Blood Cell Distribution Width and Mortality in Critically Ill Patients with Acute Kidney Injury. Biomed. Res. Int. 2018, 9658216, https://doi.org/10.1155/2018/9658216 (2018).

12. Elhosseiny, S. et al. The Value of Adding Red Cell Distribution Width to Mehran Risk Score to Predict Contrast-induced Acute Kidney Injury in Patients with Acute Coronary Syndrome. Cureus 10, e2911, https://doi.org/10.7759/cureus.2911 (2018).

13. Johnson, A. E. et al. MIMIC-III, a freely accessible critical care database. Sci. Data 3, 160035, https://doi.org/10.1038/sdata.2016.35 (2016).

14. Mehta, R. L. et al. Acute Kidney Injury Network: report of an initiative to improve outcomes in acute kidney injury. Crit. Care 11, R31, https://doi.org/10.1186/cc5713 (2007).

15. Group, K. D. I. G. O. K. A. K. I. W. KDIGO clinical practice guideline for acute kidney injury. Kidney Int, Suppl. 2, 1-138 (2012).

16. McCullough, P. A. et al. ABT-719 for the Prevention of Acute Kidney Injury in Patients Undergoing High-Risk Cardiac Surgery: A Randomized Phase 2b Clinical Trial. J. Am. Heart Assoc. 5, e003549, https://doi.org/10.1161/jaha.116.003549 (2016).

17. Cuartero, M., Betbese, A. J., Nunez, K., Baldira, J. \& Ordonez-Llanos, J. Does Whole-Blood Neutrophil Gelatinase-Associated Lipocalin Stratify Acute Kidney Injury in Critically Ill Patients? Dis. Markers 2019, 8480925, https://doi.org/10.1155/2019/8480925 (2019).

18. Lin, K., Hu, Y. \& Kong, G. Predicting in-hospital mortality of patients with acute kidney injury in the ICU using random forest model. Int. J. Med. Inf. 125, 55-61, https://doi.org/10.1016/j.ijmedinf.2019.02.002 (2019).

19. Zhang, Z., Zhu, C., Mo, L. \& Hong, Y. Effectiveness of sodium bicarbonate infusion on mortality in septic patients with metabolic acidosis. Intensive Care Med. 44, 1888-1895, https://doi.org/10.1007/s00134-018-5379-2 (2018).

20. Quan, H. et al. Coding algorithms for defining comorbidities in ICD-9-CM and ICD-10 administrative data. Med. Care 43, $1130-1139$ (2005).

21. Knaus, W. A. et al. The APACHE III prognostic system. Risk prediction of hospital mortality for critically ill hospitalized adults. Chest 100, 1619-1636 (1991).

22. Le Gall, J. R. et al. The Logistic Organ Dysfunction system. A new way to assess organ dysfunction in the intensive care unit. ICU Scoring Group. JAMA 276, 802-810 (1996).

23. Vincent, J. L. et al. The SOFA (Sepsis-related Organ Failure Assessment) score to describe organ dysfunction/failure. On behalf of the Working Group on Sepsis-Related Problems of the European Society of Intensive Care Medicine. Intensive Care Med. 22, 707-710 (1996).

24. Johnson, A. E. \& Kramer, A. A. \& Clifford, G. D. A new severity of illness scale using a subset of Acute Physiology And Chronic Health Evaluation data elements shows comparable predictive accuracy. Crit. Care Med. 41, 1711-1718, https://doi.org/10.1097/ CCM.0b013e31828a24fe (2013).

25. Bone, R. C. et al. Definitions for sepsis and organ failure and guidelines for the use of innovative therapies in sepsis. The ACCP/ SCCM Consensus Conference Committee. American College of Chest Physicians/Society of Critical Care Medicine. Chest 101, 1644-1655 (1992).

26. Wasilewski, J. et al. Prognostic value of red blood cell distribution width in patients with left ventricular systolic dysfunction: Insights from the COMMIT-HF registry. Cardiol. J. 25, 377-385, https://doi.org/10.5603/CJ.a2017.0037 (2018).

27. Nam, J. S., Ahn, C. W., Kang, S., Kim, K. R. \& Park, J. S. Red Blood Cell Distribution Width Is Associated with Carotid Atherosclerosis in People with Type 2 Diabetes. J. Diabetes Res. 2018, 1792760, https://doi.org/10.1155/2018/1792760 (2018).

28. Zbierska-Rubinkiewicz, K. et al. Creatine kinase-MB and red cell distribution width as predictors of contrast-induced nephropathy after percutaneous coronary intervention in acute myocardial infarction. Folia Med. Cracov 57, 87-99 (2017).

29. Akin, F. et al. Relation of red cell distribution width to contrast-induced acute kidney injury in patients undergoing a primary percutaneous coronary intervention. Coron. Artery Dis. 26, 289-295, https://doi.org/10.1097/mca.0000000000000223 (2015).

30. Cho, A. Y., Yoon, H. J., Lee, K. Y. \& Sun, I. O. Clinical characteristics of sepsis-induced acute kidney injury in patients undergoing continuous renal replacement therapy. Ren. Fail. 40, 403-409, https://doi.org/10.1080/0886022x.2018.1489288 (2018).

31. Vashistha, T. et al. Red Cell Distribution Width and Mortality in Hemodialysis Patients. Am. J. Kidney Dis. 68, 110-121, https://doi. org/10.1053/j.ajkd.2015.11.020 (2016).

32. Zhang, T., Li, J., Lin, Y., Yang, H. \& Cao, S. Association Between Red Blood Cell Distribution Width and All-cause Mortality in Chronic Kidney Disease Patients: A Systematic Review and Meta-analysis. Arch. Med. Res. 48, 378-385, https://doi.org/10.1016/j. arcmed.2017.06.009 (2017).

33. Odutayo, A. et al. AKI and Long-Term Risk for Cardiovascular Events and Mortality. J. Am. Soc. Nephrol. 28, 377-387, https://doi. org/10.1681/asn.2016010105 (2017).

34. Oh, H. J. et al. Red blood cell distribution width is an independent predictor of mortality in acute kidney injury patients treated with continuous renal replacement therapy. Nephrol. Dial. Transpl. 27, 589-594, https://doi.org/10.1093/ndt/gfr307 (2012). 
35. Mizuno, A., Ohde, S., Nishizaki, Y., Komatsu, Y. \& Niwa, K. Additional value of the red blood cell distribution width to the Mehran risk score for predicting contrast-induced acute kidney injury in patients with ST-elevation acute myocardial infarction. J. Cardiol. 66, 41-45, https://doi.org/10.1016/j.jjcc.2014.09.006 (2015).

36. Jia, L., Zhang, W., Jia, R., Zhang, H. \& Chen, X. Construction Formula of Biological Age Using the Principal Component Analysis. Biomed. Res. Int. 2016, 4697017, https://doi.org/10.1155/2016/4697017 (2016).

37. Doyle, J. F. \& Forni, L. G. Acute kidney injury: short-term and long-term effects. Crit. Care 20, 188, https://doi.org/10.1186/s13054016-1353-y (2016).

38. Pekkarinen, P. T. et al. Association of extracerebral organ failure with 1-year survival and healthcare-associated costs after cardiac arrest: an observational database study. Crit. Care 23, 67, https://doi.org/10.1186/s13054-019-2359-z (2019).

39. Hagen, M. et al. Systemic inflammatory response syndrome and long-term outcome after intracerebral hemorrhage. Neurol. Neuroimmunol. Neuroinflamm 6, e588, https://doi.org/10.1212/nxi.0000000000000588 (2019)

40. Deswal, A. et al. Cytokines and cytokine receptors in advanced heart failure: an analysis of the cytokine database from the Vesnarinone trial (VEST). Circulation 103, 2055-2059 (2001).

41. Semba, R. D. et al. Serum antioxidants and inflammation predict red cell distribution width in older women: the Women's Health and Aging Study I. Clin. Nutr. 29, 600-604, https://doi.org/10.1016/j.clnu.2010.03.001 (2010).

42. Rabb, H. et al. Inflammation in AKI: Current Understanding, Key Questions, and Knowledge Gaps. J. Am. Soc. Nephrol. 27, 371-379, https://doi.org/10.1681/asn.2015030261 (2016).

43. Pavlakou, P., Liakopoulos, V., Eleftheriadis, T., Mitsis, M. \& Dounousi, E. Oxidative Stress and Acute Kidney Injury in Critical Illness: Pathophysiologic Mechanisms-Biomarkers-Interventions, and Future Perspectives. Oxid. Med. Cell Longev. 2017, 6193694, https://doi.org/10.1155/2017/6193694 (2017).

44. Ghaffari, S. Oxidative stress in the regulation of normal and neoplastic hematopoiesis. Antioxid. Redox Signal. 10, 1923-1940, https://doi.org/10.1089/ars.2008.2142 (2008).

\section{Acknowledgements}

We would like to thank all the researchers who built and maintained the MIMIC-III database. At the same time, we appreciated all patients who agreed to offer the data to the MIMIC-III database. The study was supported by grants from Wu Jieping Medical Foundation Clinical Research Funding (No. 320.6750.16050) and Scientific Research Found of Capital Medical University (PYZ2018054).

\section{Author contributions}

Linpei Jia extracted data from MIMIC-III database. Linpei Jia, Rufu Jia and Jingyan Yang finished the statistical analysis. Hongliang Zhang and Shijun Cui wrote the manuscript. Hongliang Zhang, Qiang Jia and Lixiao Hao revised the manuscript. All authors have read and approved the final version.

\section{Competing interests}

The authors declare no competing interests.

\section{Additional information}

Supplementary information is available for this paper at https://doi.org/10.1038/s41598-020-61516-y.

Correspondence and requests for materials should be addressed to L.J., R.J. or H.Z.

Reprints and permissions information is available at www.nature.com/reprints.

Publisher's note Springer Nature remains neutral with regard to jurisdictional claims in published maps and institutional affiliations.

Open Access This article is licensed under a Creative Commons Attribution 4.0 International License, which permits use, sharing, adaptation, distribution and reproduction in any medium or format, as long as you give appropriate credit to the original author(s) and the source, provide a link to the Creative Commons license, and indicate if changes were made. The images or other third party material in this article are included in the article's Creative Commons license, unless indicated otherwise in a credit line to the material. If material is not included in the article's Creative Commons license and your intended use is not permitted by statutory regulation or exceeds the permitted use, you will need to obtain permission directly from the copyright holder. To view a copy of this license, visit http://creativecommons.org/licenses/by/4.0/.

(c) The Author(s) 2020 